\title{
Safety of live attenuated Japanese encephalitis vaccine given at the age of 9 months in National Immunisation Programme of Sri Lanka
}

\author{
K Sanchayan ${ }^{1}$, R Fernandopulle ${ }^{2}$, A Amarasinghe ${ }^{3}$, S N Thiyahiny ${ }^{1}$, S Sri Ranganathan ${ }^{4}$ \\ (Index words: adverse events following immunisation, cohort event monitoring, live attenuated Japanese en- \\ cephalitis vaccine, vaccine safety)
}

\begin{abstract}
Objectives This study was designed to describe the safety profile of the single dose live attenuated Japanese encephalitis vaccine (LJEV) SA-14-14-2 given at the age of 9 months in the National Immunisation programme of Sri Lanka.
\end{abstract}

Methods A cohort event monitoring (CEM) was conducted in the Jaffna district during November 2012 to January 2015. A representative sample of 3041 infants who received the LJEV at the age of 9 months was followed up actively over telephone interviews on days 1, 3, 14, 30 and 45 for adverse events (AE). Parents were encouraged to self-report in between these interviews. When an $A E$ is notified, further clinical information was obtained through in-depth interviews and home/hospital visits to determine as an adverse event following immunisation (AEFI). Investigators independently reviewed each AEFI for consistent causal association with LJEV according to WHO causality assessment.

Results Of the 2878 (94\%) infants who completed the follow up of 14 days, 911 (32\%) experienced 1423 AEFIs. Of them, 376 (26\%) were identified as AEFI with consistent causal association to LJEV (AEFIc). Irritability (53/ 1000 doses administered) and fever $\geq 100.4^{\circ} \mathrm{F}(46 / 1000$ doses administered) accounted for $41 \%$ and $35 \%$ of AEFIcrespectively. Majority of AEFI (940) were identified as inconsistent as there were alternate causes. Nineteen AEFIc in 14 infants were classified as serious since they led to hospitalisation. Of the 2392 (79\%) infants who completed 45 days follow up, 1022 experienced 1804 AEFI during $15-45$ days. Only 20 were identified as AEFIc. There were no reported fatal or life threatening AEs.

Conclusions LJEV administered to infants at 9 months was devoid of any significant safety concerns as most of the AEFIs were non-serious and resolved completely. CEM is a useful method for AEFI surveillance.

Ceylon Medical Journal 2016; 61: 99-105

DOI: http://doi.org/10.4038/cmj.v61i3.8344

\section{Introduction}

Serious nature of the Japanese encephalitis (30\% case fatality rate, $30-50 \%$ residual neurological deficits), absence of effective treatment, and the disease burden were the decisive factors for incorporating mouse brain derived inactivated Japanese encephalitis (MBDJE) vaccine in the National Immunisation Programme (NIP) of Sri Lanka in 1988 [1, 2]. The MBDJE vaccine was replaced by the single dose live attenuated Japanese encephalitis (SA-14-14-2) vaccine (LJEV) in 2009 [2, 3]. Initially LJEV was given at the age of 1 year, then advanced to 9 months in 2011 and reverted back to 1 year in April 2015.

Effectiveness of an immunisation programme mainly depends on the efficacy and safety of the vaccine and its expected high coverage of target population. For example, the MBDJE vaccine could not be sustained in the programme because of its safety, cost and feasibility constraints. Safety concerns were raised after a report released in 1995 suggesting a temporal relationship of the vaccine to acute disseminated encephalomyelitis [4]. Cost and feasibility constraints were mainly related to its four doses schedule (primary - day 0, day 30, boosters 1 and 4 years apart). This prompted replacing it with LJEV.

Efficacy of vaccines especially during pre-marketing clinical trials is generally measured by their immunogenicity. Short and long term (1 month-5years) immunogenicity of the LJEV had been documented in studies

${ }^{1}$ Department of Pharmacology, Faculty of Medicine, University of Jaffna, ${ }^{2}$ General Sir John Kotelawala Defence University, Kandawala Estate, Rathmalana, ${ }^{3}$ Epidemiology Unit, Ministry of Health, Colombo and ${ }^{4}$ Department of Pharmacology, Faculty of Medicine, University of Colombo, Sri Lanka.

Correspondence: KS, e-mail: <skumuthini79@yahoo.com>. Received 18 April and revised version accepted 14 May 2016.

This is an open-access article distributed under the terms of the Creative Commons Attribution License, which permits unrestricted use, distribution, and reproduction in any medium, provided the original author and source are credited. 
before it was introduced into our NIP [5-9]. Coverage was anticipated to be high, as it was only one dose. Some studies have looked at the safety of this vaccine and reported reasonable safety profile [6, 7, 10-13]. However, for documentation of safety, data from post-marketing surveillance when the vaccine is actually used in the programme are vital, and responsibility of generating such data lies with the countries which have incorporated these vaccines in their NIP.

In the case of LJEV, two post-marketing surveillance studies in China have reported adverse events following immunisation (AEFI) with an incidence of 61.2 and 96.55 per million doses in infants aged 8 months to 6 years [10, 13]. Both studies used the AEFI data generated from the routine post-marketing surveillance programmes using spontaneous reporting system (SRS). It is well known that under-reporting is a major limitation in SRS: For example, only less than $10 \%$ of all serious and $2-4 \%$ of non-serious suspected adverse drug reactions are ever reported spontaneously $[14,15]$. This problem of under-reporting in SRS has been highlighted in a study from Sri Lanka which utilised cohort event monitoring (CEM) study design to study the safety of MBDJE vaccine [3].

We carried out this study to describe safety of LJEV when it was being given in the NIP at the age of 9 months. We have employed CEM study design to overcome the limitations of SRS. We believe that the data generated through the active CEM study design will complement the existing data on safety of LJEV, both quantitatively and qualitatively.

\section{Methods}

This prospective observational cohort event monitoring study in infants who received their routine LJEV at the age of 9 months was carried out between November 2012 and December 2014. This study was conducted in Jaffna Regional Directorate of Health Services (RDHS) area. It is located in the Northern Province of Sri Lanka and has a population of 610640 [16], which is about $2.8 \%$ of the total population [17].

The sample size was calculated as 3000 , according to WHO recommendations, to give a $95 \%$ probability of identifying a minimum of one adverse event (AE) occurring at the rate of more than 1:1000 [18]. Proportionate stratified sampling was employed to recruit a representative cohort from the Medical Officer of Health $(\mathrm{MOH})$ areas [19]. For administration of public health services, each RDHS area in Sri Lanka is divided into $\mathrm{MOH}$ areas. To improve the efficiency and cost-effectiveness of recruitment, within each $\mathrm{MOH}$ area, the key immunisation clinics were selected by going through the previous immunisation registers and discussing with the field public health staff.

The selected clinics were visited on the day of administration of LJEV. Parent or guardian of the infants were approached while they were waiting for routine procedures. After explaining the purpose of the study, the information sheet was distributed to each of them. Adequate time was given to read the information sheet and to clarify any concerns if present. Non-residents of Jaffna RDHS area, parents or primary caregiver who are unreachable by phone and infants who received the LJEV after the age of 1 year were excluded. Informed written consent was obtained from the eligible parents or guardian to recruit their infants into the cohort. The importance of follow up was explained to them and an event recall diary was given. They were instructed to record all the adverse events during the follow up period. Background information and contact telephone numbers were obtained. They were trained to measure, read and interpret axillary temperature of their infants and also supplied with a mercury thermometer. Parents / guardians were also informed that they could call the principal investigator in case of any AEs or for any clarification.

Each infant in the cohort was followed up actively for 45 days by interviewing the parent or primary caregiver over the phone on days 1, 3, 14, 30 and 45. At each interview, general well-being of the infant, occurrence of any AEs (with a check list) and visits to a doctor or hospital were documented. In case of any AE, further details were obtained through in-depth interviews. In addition, PI visited the infants at home or hospital to collect further clinical information if deemed necessary. Parents were also encouraged to self-report AEs or concerns to the PI over the phone. In instances where the parent/primary caregiver was not contactable over the phone, assistance of the public health midwife PHM) was sought to contact the family. When this too failed, the $\mathrm{PHM} / \mathrm{MOH}$ records were reviewed to see whether there had been any significant AEFI or death during the study period. Specifically designed pre-tested interviewer administered structured questionnaires were used to collect data at the various points from recruitment to end of follow up.

Data from the questionnaires were transferred to a custom-designed Microsoft Access database for easy retrieval and further analysis. The WHO defines an AEFI as any untoward medical occurrence which follows immunisation and which does not necessarily have a causal relationship with the usage of vaccine [20]. As per this definition, the adverse events reported by the parents, which were further corroborated by in depth telephone interview, home or hospital visits, or by review of clinical records were considered as AEFI.Two investigators reviewed all these AEFIs to identify individual AEFIs such as fever, injection site reactions, anaphylaxis, etc. using universal guidelines such as Brighton collaboration case definitions [20] or WHO - adverse reaction terminology as appropriate [21]. The processed data were used in subsequent causality analysis of AEFI.

Two investigators independently assessed the causality of the AEFIs using the WHO work sheet for 
AEFI causality assessment. This worksheet had been developed using the key causality criteria such as absence of strong evi-dence for alternative causes, known causal association with vaccine or vaccination, and occurrence of AEFI within the time window of increased risk [22]. Based on causality assessment, the AEFIs were assessed to be i) consistent with causal association to immunisation ii) indeterminate iii) inconsistent with causal association to immunisation iv) unclassifiable. If there was a disagreement in the AEFI causality assessment between the investigators a consensus decision was arrived following a discussion. AEFI which have consistent causal association to LJEV (AEFIc) were further classified into serious and non-serious based on CIOMS/WHO definition for serious AEFI [20]. This study was approved by the Ethics review committee of Faculty of Medicine, University of Colombo (EC/12-089).

\section{Results}

Since previous studies have reported that majority of vaccine related AEs occur within 14 days of LJEV administration, we analysed the AEs which occurred in 0-14 days separately from those occurred in the next 15-45 days [8, 23].

\section{AEFI reported during 0-14 days of follow up}

Of the ( $n=3379)$ infants screened, 3041 (90\%) satisfied the inclusion criteria and they were recruited into the cohort after obtaining informed consent from the mother or guardian: 2878 of the 3041 infants (94\%) were followed up successfully up to 14 days. Mean age was 9 months and 10 days and 51\% were males. During this follow up, 1423 AEFI were reported in 911 infants with a rate of 1.6 AEFI/ infant who experienced any AEFI (Figure 1). This gave the incidence of children experienced AEFI as 32 per 100 immunisation. The overall rate of AEFI was 0.5 / infant in all who received JE vaccine (1423 AEFI / 2878 Infants).

Of the 1423 AEFI,376 reported in 335 infants were assessed as AEFIc (Table 1). Irritability (41\%) was the commonest AEFIc reported in 153 infants (53 per 1000 doses administered). 85\% of irritability was reported within 24 hours of immunisation and $92 \%$ recovered on the same day. Mothers perceived that it was associated with the pain or fever following immunisation. Fever $\geq 100^{\circ} \mathrm{F}$ was reported in 133 infants (46 per 1000 doses administered): $39 \%$ of infants recovered in one day and another $44 \%$ recovered in the next 48 hours, 50 infants sought medical advice and 6 were hospitalised. Those who were hospitalised had few additional symptoms.

Of the 1423 AEFIs, 940 (66\%) were assessed as inconsistent with causal association to LJEV (coincidental). Majority of them were assigned to this group because there was clinical evidence of alternative cause for the adverse event. Hence the causal relationship to LJEV was assessed to be inconsistent. Examples included upper respiratory tract and gastrointestinal infections with similar symptoms seen in one or more children in the family. As per the WHO worksheet, 107 AEFIs (7.5\%) were assigned to indeterminate because of insufficient information or conflicting trends.

NineteenAEFIc reported in 14 infants were assessed as serious. All but one were classified as serious because the infants were hospitalised (mean hospital stay was two days) (Table 2). All infants with serious AEFIc recovered completely.

\section{AEFI reported during 15-45 days}

Of the 3041 recruited infants, 2392 (79\%) were followed up until 45 days. Their mean age was 9 months and 10 days and 51\% were males. During 15-45 days, 1804 AEFI were reported in 1022 infants (1.8 AEFI/ infant) which amounted to 42 infants experiencing AEFIs per 100 immunisation. However, only 20 (1.1\%) of these AEFIs reported in 19 infants were assessed as AEFIc, with one being classified as serious AEFIc due to hospitalisation (Table 1, 2). There were no deaths or life threatening AEFIs during the entire follow up period of 45 days.

\section{Discussion}

The results of this prospective active pharmacovigilance study where a large number of infants were followed up intensively for 45 days has provided evidence that the LJEV is relatively safe and devoid of any fatal or life threatening AEFI. This evidence has strengthened the existing knowledge about the safety of this vaccine obtained via other study designs [5-8, 10-13, 23]. Our study further support the previously published evidence of that the majority AEFI for LJEV occur during the first 14 days following immunisation $[8,23]$. In addition, this study documents safety of LJEV when it is given at the age of 9 months.

In our study, hospitalisation was the leading reason for an AEFIc to be classified as serious. Hospitalisation criteria is more likely to reflect the seriousness of the event in adults. However, in infants, hospitalisation does not necessarily reflect seriousness of the event as it is heavily influenced by the parental anxiety and health seeking behaviour.

Irritability was reported as a common AEFIc, however, there was no standard case definition for irritability as an AEFI. It was a subjective event reported by mother/ primary caregiver when they witnessed the infant not to be engaged in usual activities, cried with no obvious reason or refused solid foods. In most of the instances, it was the only event and infants recovered completely within 24 hours. In our study, there were no reports of persistent crying which was continuous and unaltered for more than 3 hours [24]. 
After causality assessment, occurrence of fever $\geq 100.4^{\circ} \mathrm{F}$ was determined to be 46 per 1000 doses in our study. In vaccine pharmacovigilance, the researchers have found difficulties in attributing fever to the vaccine studied [25, 26]. This is because fever is a very common presentation in infancy with many causes which generally remain undiagnosed in routine clinical practice. Hence, when assessing the causality, applying the "absence of an alternative cause” criterion for fever in infants is always a challenge.

Figure 1. Schematic representation of the CEM following live attenuated Japanese encephalitis vaccine during 0 -14 days

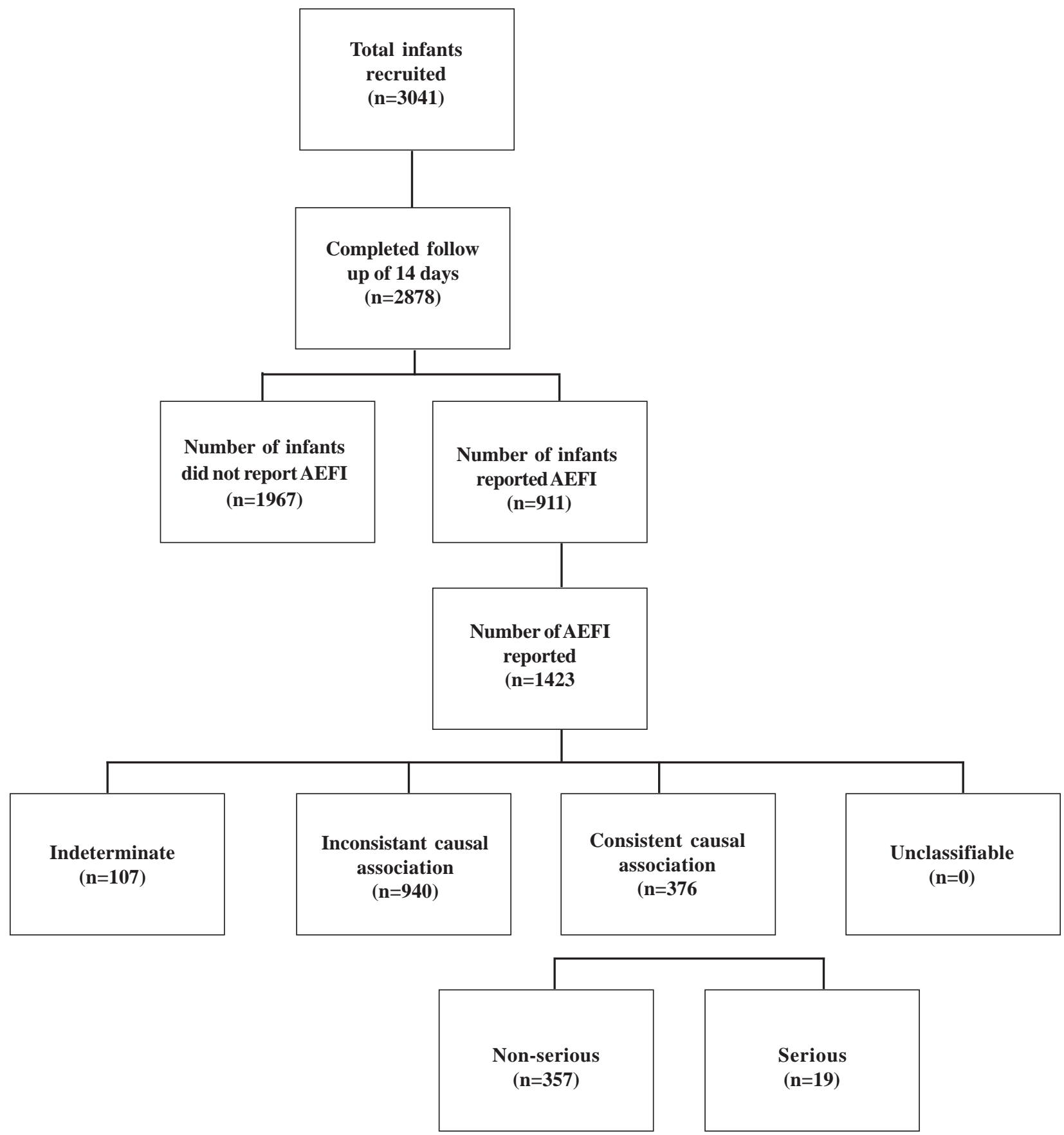


Table 1. Adverse events following immunization with consistent causal association (AEFIc) to live attenuated Japanese encephalitis vaccine detected during cohort event monitoring

\begin{tabular}{|c|c|c|c|c|}
\hline AEFIC & $\begin{array}{c}\text { Number of } \\
\text { AEFIc during } \\
\text { 0-14 days }\end{array}$ & $\begin{array}{c}\text { Rate of AEFIc } \\
\text { in 0-14 days } \\
\text { per } 1000 \text { doses } \\
\quad(n=2878)\end{array}$ & $\begin{array}{c}\text { Number of } \\
\text { AEFI cduring } \\
15-45 \text { days } \\
(n=2392)\end{array}$ & $\begin{array}{c}\text { Rate of AEFIc in } \\
\text { during } 15-45 \text { days } \\
\text { per } 1000 \text { doses }\end{array}$ \\
\hline Irritability & 153 & 53 & - & - \\
\hline Fever $100.4-102^{\circ} \mathrm{F}$ & 90 & 31 & 8 & 3.3 \\
\hline High fever $\geq 102.2^{\circ} \mathrm{F}$ & 43 & 14 & 6 & 2.5 \\
\hline Diarrhoea & 39 & 14 & 3 & 1.25 \\
\hline Vomiting & 18 & 6.3 & 1 & 0.42 \\
\hline Urticaria & 12 & 4.2 & - & - \\
\hline Local reaction at or near injection site & 8 & 2.8 & 1 & 0.42 \\
\hline Injection site induration & 4 & 1.4 & - & - \\
\hline Crying abnormal & 3 & 1.0 & - & - \\
\hline Febrile convulsion* & 5 & 1.7 & 1 & 0.42 \\
\hline Feeding problem (Reduced feeding) & 1 & 0.3 & - & - \\
\hline Total & 376 & 130.6 & 20 & 8.36 \\
\hline
\end{tabular}

*generalised convulsive seizure associated with fever

Table 2. Serious adverse events following immunization with consistent causal association (AEFIc) to live attenuated Japanese encephalitis vaccine

\begin{tabular}{|c|c|c|c|c|c|c|c|c|c|c|}
\hline \multirow[t]{2}{*}{ Type of AEFIC } & \multirow[t]{2}{*}{$\begin{array}{c}\text { Number of infants } \\
\text { with serious } \\
\text { AEFIC } \\
(n=15)\end{array}$} & \multicolumn{5}{|c|}{$\begin{array}{l}\text { Time onset of AEFIc following } \\
\text { administration of LJEV (days) }\end{array}$} & \multicolumn{4}{|c|}{$\begin{array}{l}\text { Duration of } \\
\text { hospitalisation } \\
\text { (days) } \\
(n=14)\end{array}$} \\
\hline & & $\begin{array}{c}1 \\
(n=1)\end{array}$ & $\begin{array}{c}2-3 \\
(n=6)\end{array}$ & $\begin{array}{c}4-7 \\
(n=2)\end{array}$ & $\begin{array}{l}8-14 \\
(n=5)\end{array}$ & $\begin{array}{l}>14 \\
(n=1)\end{array}$ & $\begin{array}{c}1 \\
(n=4)\end{array}$ & $\begin{array}{c}2 \\
(n=4)\end{array}$ & $\begin{array}{c}3 \\
(n=5)\end{array}$ & $\begin{array}{c}\geq 4 \\
(n=1)\end{array}$ \\
\hline Fever & 2 & - & 2 & - & - & - & 1 & - & 1 & - \\
\hline Febrile convulsions* & 6 & - & 3 & - & 2 & 1 & 1 & 2 & 2 & - \\
\hline Papular urticaria & 1 & - & 1 & - & - & - & - & 1 & - & - \\
\hline Acute Gastroenteritis** & 2 & 1 & - & - & 1 & - & 1 & - & - & 1 \\
\hline Diarrhoea & 4 & - & - & 2 & 2 & - & 1 & 1 & 2 & - \\
\hline
\end{tabular}

Note: *one febrile convulsion case was not hospitalised. ${ }^{* *}$ considered the diagnosis given in bed head ticket

The AEFI rates reported in our study was different from other studies. For example, fever following LJEV was reported in recent literature as 76.76 per million doses [13] through post marketing surveillance and 21.7 per 100 doses in a randomised trial [8]. The difference in the rates of AEFI could be due to differences in age of the participants, case definitions, causality assessment method, duration and method of follow up. The follow up rate of $94 \%$ for 14 days in our study was higher than the similar CEM studies $[27,28]$. This was possible because the families were contacted at their convenient time, option of self-reporting and the voluntary help from PHMs. 
Cohort event monitoring has the potential of identifying previously unrecognised and unsuspected AEFI [29]. As mentioned earlier, a sample size of 3000 has a $95 \%$ probability of identifying a minimum of one AE occurring at the rate of more than 1:1000 [18]. Our study did not detect any such AEs within 45 days of LJEV. From our experience, we feel that following up a larger sample for a period of 45 days or more require either a large research team or incorporation of active pharmacovigilance into the existing public health programmes.

CEM, though appears to be effective in vaccine pharma-covigilance, it has some potential challenges which we experienced during this study. First it was resource intensive because it demanded travelling to distant rural areas for recruitment and follow up. Secondly despite recruiting infants from families who were contactable over the phone, in reality, we found about one third of the families could not be reached in the given phone numbers at least in one of the follow up calls. Though we circum-vented this problem by contacting the families through PHMs, we feel this voluntary support of PHMs will not be sustainable in the long run. Thirdly, differentiating vaccine related AEs and co-incidental symptoms is a difficult task. Having a control group could have helped to overcome this issue. But for a vaccine already in the NIP, it is ethically unacceptable to have a control group which compels researchers to devise their own strategy to overcome this limitation.

\section{Conclusions}

We conclude that the LJEV administered at the age of 9 months is relatively safe. The AEFI which were assessed to be consistent with causal association to LJEV were mostly non-serious. CEM can be adapted to study safety of new vaccines in NIPs if the challenges in data collection and causality assessment could be addressed.

\section{Funding}

Faculty research grant from University of Jaffna, and research grant for local $\mathrm{PhD}$ from University Grants Commission (UGC/ICD/RG 2012/02), Sri Lanka.

\section{Acknowledgements}

We acknowledge the Chief Epidemiologist, Ministry of Health, Regional Director of Health Services (Jaffna) and Medical Officers of Health (study areas) for granting approval to collect the data. Supervising PHMs, PHMs, health volunteers and data collectors are acknowledged for their support and involvement in field work. Authors also thank Mr S. Thayaparan, Staff Technical officer, Department of Pharmacology, Faculty of Medicine, University of Jaffna for his assistance in development and maintenance of the database. We also thank the parents and guardians for their unconditional support.

\section{Conflicts of interests}

There are no conflicts of interest.

\section{References}

1. Soloman T, Dung NM, Kneen R, et al. Japanese Encephalitis. J Neurol Neurosurg Psychiatry 2000; 68: 405-15.

2. Epidemiology Unit. Japanese Encephalitis: a manual for medical officers of health Sri Lanka: Ministry of Health; 2008.

3. De Alwis KN, Abeysinghe MR, Wickramesinghe AR, Wijesinghe PR. A cohort event monitoring to determine the adverse events following administration of mouse brain derived, inactivated Japanese Encephalitis vaccine in an endemic district in Sri Lanka. Vaccine 2014; 32: 924-30.

4. Ohtaki E, Matsuishi T, Hirano Y, Maekawa K. Acute disseminated encephalomyelitis after treatment with Japanese B encephalitis vaccine (Nakayama-Yoken and Beijing strains). J Neurol Neurosurg Psychiatry 1995; 59: 316-7.

5. Gatchalian S, Yao Y, Zhou B, et al. Comparison of the immunogenicity and safety of measles vaccine administered alone or with live, attenuated Japanese encephalitis SA 14-14-2 vaccine in Philippine infants. Vaccine 2008; 26 : 2234-41.

6. Wijesinghe PR, Abeysinghe MR, Yoksan S, et al. Safety and immunogenicity of live-attenuated Japanese encephalitis SA 14-14-2 vaccine co-administered with measles vaccine in 9-month-old infants in Sri Lanka. Vaccine 2014; 32: 4751-7.

7. Sohn YM, Park MS, Rho HO, et al. Primary and booster immune responses to SA 14-14-2 Japanese encephalitis vaccine in korean infants. Vaccine 1999; 17: 2259-64.

8. Feroldi E, Pancharoen C, Kosalaraksa P, et al. Primary Immunization of Infants and Toddlers in Thailand with Japanese Encephalitis Chimeric Virus Vaccine in Comparison with SA14-14-2. Pediatr Infect Dis J 2014; 33: 643-9.

9. Sohn YM, Tandan JB, Yoksan S, et al. A 5-year follow-up of antibody response in children vaccinated with single dose of live attenuated SA14-14-2 Japanese encephalitis vaccine: immunogenicity and anamnestic responses. Vaccine 2008; 26: 1638-43.

10. Liu Y, Lin H, Zhu Q, et al. Safety of Japanese encephalitis live attenuated vaccination in post-marketing surveillance in Guangdong, China, 2005-2012. Vaccine 2014; 32: 1768-73.

11. Liu Z-L, Hennessy S, Strom BL, et al. Short-Term Safety of Live Attenuated Japanese Encephalitis Vaccine (SA1414-2): Results ofa Randomized Trial with 26,239 Subjects. J Infect Dis 1997; 176: 1366-9.

12. Ranganath BG. Incidence of adverse events following immunization with SA14-14-2 Japanese Encephalitis vaccine among children of 6-10 years in Kolar, India. J Clin Biomed Sci 2011; 1: 49-54. 
13. Wang Y, Dong D, Cheng G, et al. Post-marketing surveillance of live-attenuated Japanese encephalitis vaccine safety in China. Vaccine 2014; 32: 5875-9.

14. Rawlins MD. Pharmacovigilance: paradise lost, regained or postponed? J R Coll Physicians Lond 1995; 29: 41-9.

15. Smith CC, Bennett PM, Pearce HM, et al. Adverse drug reactions in a hospital general medical unit meriting notification to the Committee on the Safety of Medicines. Br J Clin Pharmacol 1996; 42: 423-9.

16. Statistical hand book 2013: RDHS Jaffna; 2013.

17. Census of Population and housing. Sri Lanka: Department of census and statistics; 2012.

18. World Health Organization. A practical handbook on the pharmacovigilance of antiretroviral medicines Geneva: World Health Organization; 2009.

19. Vaus DDD. Survey in Social Research. 3rd ed. Australia: Allen \& Unwin Pty Ltd; 1991.

20. CIOMS. Definition and Application of Terms for Vaccine Pharmacovigilance: Report of CIOMS/WHO Working Group on Vaccine Pharmacovigilance Geneva 2012. Available from: http://www.who.int/vaccine_safety/ initiative/tools/CIOMS_report_WG_vaccine.pdf.

21. World Health Organization. WHO Adverse Reaction Terminology 2008.

22. World Health Organization. causality assassment of an adverse event following immunization (AEFI) user manual for the revised WHO classification. Geneva: World Health Organization; 2013.
23. Kim DS, Houillon G, Jang GC, et al. A randomized study of the immunogenicity and safety of Japanese encephalitis chimeric virus vaccine (JE-CV) in comparison with SA1414-2 vaccine in children in the Republic of Korea. Hum Vaccin Immunother 2014; 10: 2656-63.

24. Bonhoeffer J, Vermeer P, Halperin S, et al. Persistent crying in infants and children as an adverse event following immunization: case definition and guidelines for data collection, analysis, and presentation. Vaccine 2004; 22: 586-91.

25. Michael Marcy S, Kohl KS, Dagan R, et al. Fever as an adverse event following immunization: case definition and guidelines of data collection, analysis, and presentation. Vaccine 2004; 22: 551-6.

26. Kohl KS, Marcy SM, Blum M, et al. Fever after immunization: current concepts and improved future scientific understanding. Clin Infect Dis 2004; 39: 389-94.

27. Dodoo AN, Fogg C, Nartey ET, et al. Profile of adverse events in patients receiving treatment for malaria in urban Ghana: a cohort-event monitoring study. Drug Saf 2014; 37: 433-48.

28. Dodoo AN, Renner L, van Grootheest AC, et al. Safety Monitoring of a New Pentavalent Vaccine in the Expanded Programme on Immunisation in Ghana. Drug Saf 2007; 30: 347-56.

29. World Health Organization. The safety of medicines in public health programmes. Geneva: World Health Organization; 2006. 